- Original Article

\title{
Metoclopramide for Milk Production in Lactating Women: A Systematic Review and Meta-Analysis
}

\author{
Nik Hazlina Nik Hussain ${ }^{1}$, Norhayati Mohd Noor ${ }^{2}$, Shaiful Bahari Ismail ${ }^{2}$, Nur Amirah Zainuddin ${ }^{1}, Z^{2}$ aharah Sulaiman ${ }^{1, *}$ \\ 'Women's Health Development Unit and ${ }^{2}$ Department of Family Medicine, School of Medical Sciences, Health Campus, Universiti Sains Malaysia, \\ Kubang Kerian, Malaysia
}

Background: Breastfeeding is recognized as the optimal form of nutrition for the physical and neurological development of infants and is considered the most significant way to prevent child mortality. This study aimed to assess the effectiveness of metoclopramide for enhancing milk production in lactating women.

Methods: We searched the Cochrane Central Register of Controlled Trials and MEDLINE for randomized controlled trials comparing metoclopramide with a placebo, no treatment, or other galactagogue drugs. We included breastfeeding women with term or preterm infants.

Results: We retrieved 164 records from our search of the electronic databases and 20 records from other sources. Eight trials involving 342 lactating women that used metoclopramide were included in this review after assessing the eligibility criteria. The meta-analysis of these trials revealed that metoclopramide did not increase the milk volume of the intervention groups compared to that of the control groups. There was a significant increase in the serum concentrations of prolactin when the mothers were administered metoclopramide. No significant adverse events were reported.

Conclusion: Metoclopramide did not improve milk production in lactating women. Therefore, we do not recommend using metoclopramide to increase milk production in lactating women.

Keywords: Breast Feeding; Lactation; Galactagogues; Metoclopramide

Received: October 18, 2020, Revised: December 8, 2020, Accepted: December 17, 2020

*Corresponding Author: Zaharah Sulaiman https://orcid.org/0000-0002-4023-1845

Tel: +60-9-7676886, Fax: +60-9-7653370, E-mail: zaharah@usm.my 


\section{INTRODUCTION}

Breastfeeding is the accepted, optimal form of nutrition for the physical and neurological development of infants, and is considered the most significant way to prevent child mortality. Indeed, it has been estimated that interventions to promote breastfeeding could prevent $13 \%$ of all deaths in children $<5$ years old in developing countries. ${ }^{1)}$ Therefore, the World Health Organization (WHO) recommends the exclusive breastfeeding of children for the first 6 months of life to achieve optimal growth. ${ }^{2)}$

Although the baby-friendly hospital initiative established by the WHO and United Nation International Children's Emergency Fund (UNICEF) in 1991 has improved support for breastfeeding initiation in hospitals, similar community support has not been observed. ${ }^{3)}$ Many women initiate breastfeeding, but do not continue for the length of time recommended by the WHO. They tend to breastfeed for a shorter duration because of the perception of having an inadequate milk supply. ${ }^{4,5)}$ This misconception is commonly caused by a lack of awareness of the normal physiology of lactation. ${ }^{6}$ )

Nevertheless, consideration must also be given to the fact that lactation is a symbiotic process between mothers and infants, which can be affected by numerous factors such as maternal and neonatal health, medical conditions, improper breastfeeding techniques, breastfeeding attachment, feeding frequency, psychological state of the mother (e.g., stress and confidence levels), and pain (including painful nipples). ${ }^{7)}$

It is recommended that practitioners evaluate and improve feeding practices to facilitate the use of proper and correct techniques to manage low milk supply in women with healthy infants. ${ }^{7,8)}$ When nonpharmacological measures fail to satisfactorily addressed milk production challenges, galactagogues can be the next alternative to facili- tate increasing milk supply. ${ }^{8)}$ Galactagogues are drugs that stimulate and sustain adequate milk production ${ }^{9)}$ and can be categorized into two groups: pharmacological agents and natural products, such as fenugreek. ${ }^{9)}$ Pharmacological galactagogues are commonly prescribed by practitioners.

Metoclopramide is one such drug option, and clinical data shows its efficacy in promoting milk production in women. ${ }^{9)}$ Studies in developing countries have reported that metoclopramide enhances lactation in women who have experienced a severe decrease in milk supply. ${ }^{10)}$ However, there is some controversy surrounding the use of metoclopramide because of its adverse events, such as maternal depression and tardive dyskinesia. ${ }^{11)}$

Metoclopramide is a dopamine antagonist that increases prolactin levels, thereby initiating or augmenting milk production ${ }^{9)}$ and has been used off-label by practitioners. Although practitioners tend to use domperidone rather than metoclopramide presently because of safety issues, metoclopramide is still the drug of choice in certain countries, especially developing countries, as it is cheaper. While the efficacy and adverse events of metoclopramide have been examined in studies with large samples, the effectiveness and safety of the drug continue to be debated and, therefore, should be explored further. In this review, we assess the effectiveness of metoclopramide for milk production in lactating women.

\section{METHODS}

\section{Eligibility Criteria}

We considered only randomized controlled trials (RCTs) that compared metoclopramide with a placebo, no treatment, or other galactagogues. We excluded quasi-randomized trials from the assessment

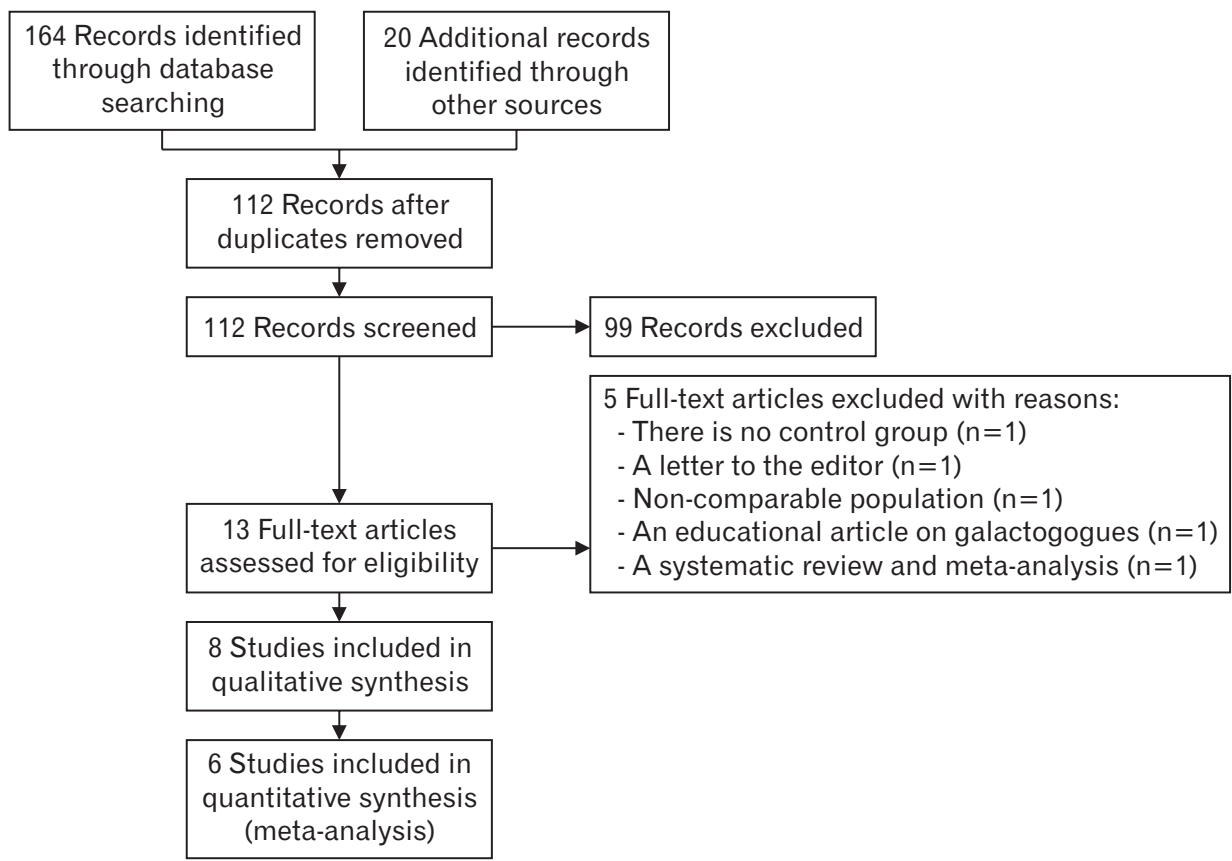

Figure 1. Study flow diagram. 


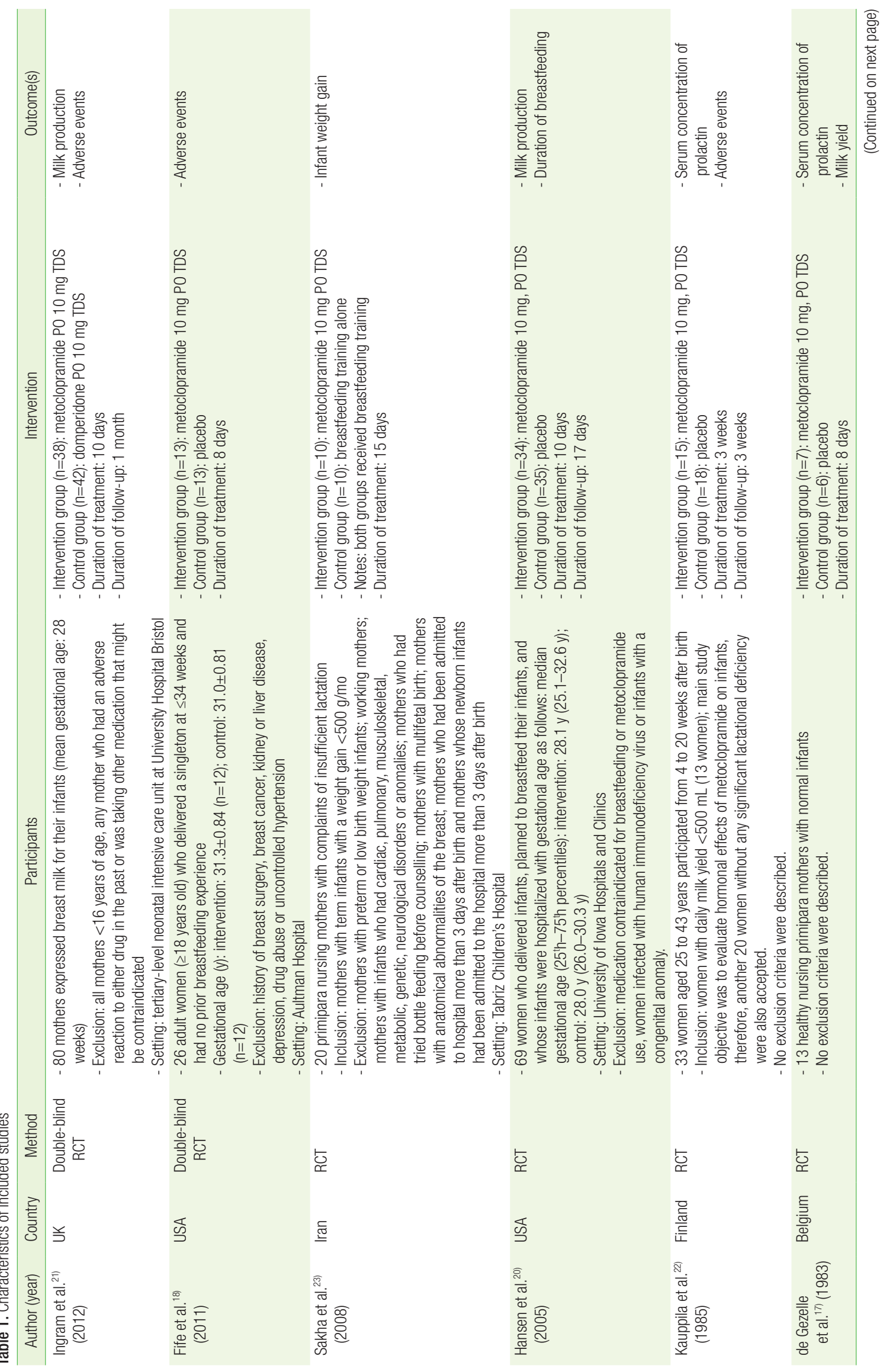



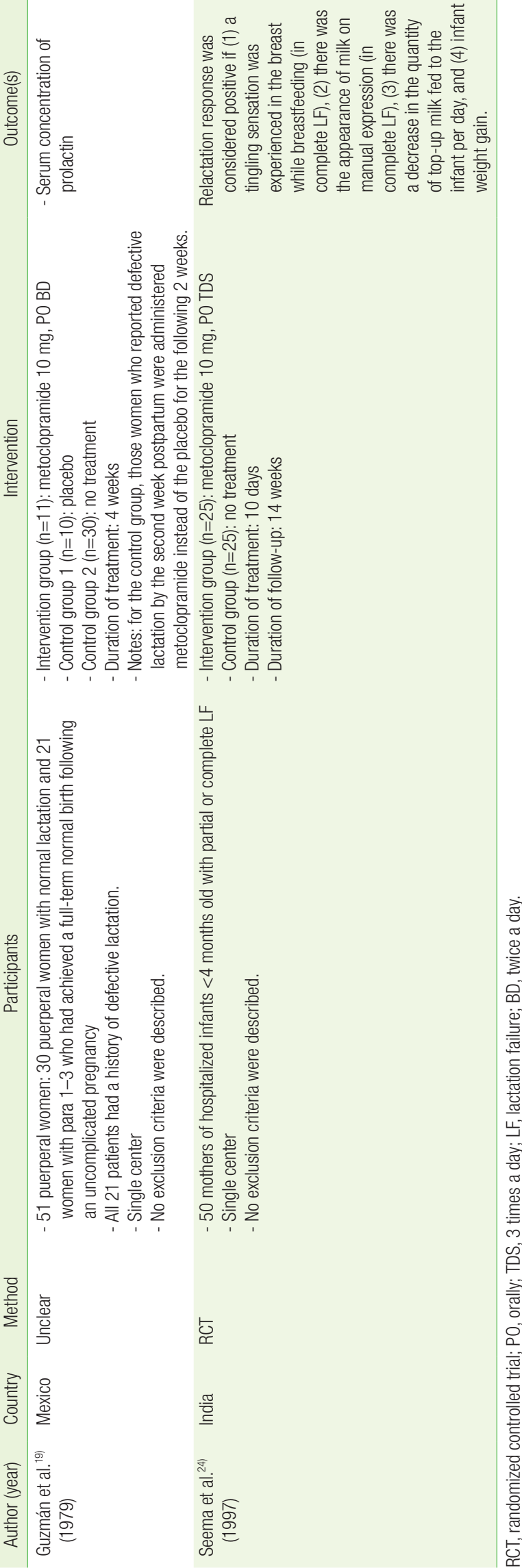

and included breastfeeding women with term or preterm infants who wanted to increase their milk production or who were having lactation problems. The intervention group treatment in each study included oral administration of metoclopramide $10 \mathrm{mg}$ at any frequency and for any duration. The comparison groups received placebos or other galactagogues such as domperidone, or no treatment.

Milk production was assessed by measuring the volume of expressed breast milk over at least 7 days and up to 14 days from the start of administration of the medication or the placebo. The effects of the intervention on milk production were observed for the duration of at least 7 days. The follow-up period for primary outcomes was between 1 and 2 weeks.

\section{Search Strategy}

We searched the Cochrane Central Register of Controlled Trials (CENTRAL 2019, Issue 4), MEDLINE, and EMBASE (till April 2019) for the words "breastfeeding," "lactation," "galactagogue," and "metoclopramide." We also checked the reference lists of all related studies for additional references to find unpublished trials or trials not identified through our electronic searches.

\section{Trial Selection}

We scanned the titles and abstracts independently of the searches and obtained full-text articles, which appeared to meet the eligibility criteria or when there was insufficient information to assess eligibility. We independently assessed the eligibility of the trials and documented any reasons for exclusion. We resolved any disagreements between the reviewing authors through discussion and contacted the article authors where clarification was needed.

\section{Data Extraction}

We prepared a data extraction form to facilitate our data extraction. For each selected study, we extracted the study setting, participant characteristics (age and obstetric history), methods (number of participants randomized and analyzed and duration of follow-up), metoclopramide dosage and frequency of administration, volume of milk expressed, maternal serum prolactin levels, infant weight gain, duration of breastfeeding, and the occurrence of adverse events.

Any disagreements were resolved through discussion. The primary outcomes were milk production and serum concentrations of prolactin. These were measured after 7 and for up to 14 days following the start of the intervention. The secondary outcomes were infant weight gain, total duration of exclusive, predominant or partial breastfeeding, and maternal adverse events due to the medication.

\section{Risk of Bias Assessment}

Risk of bias was assessed based on (1) random sequence generation, (2) allocation concealment, (3) the blinding of participants and personnel, (4) blinding of the outcome assessors, (5) completeness of the outcomes data, (6) selectivity of the outcomes reporting, and (7) other forms of bias as discussed in the Cochrane Handbook for Systematic 
Reviews of Interventions. ${ }^{12)}$ We categorized the trials as having a low, unclear, or high risk of bias across all domains.

\section{Statistical Analysis}

All statistical analyses were performed using Review Manager ver. 5.3 software (RevMan; Cochrane, London, UK). We reported categorical outcomes using risk ratios (RR), 95\% confidence intervals (CI), and numerical outcomes as mean differences (MD) and 95\% CI. We examined the heterogeneity using both fixed effects and random effects models depending on the degree of significant clinical or statistical heterogeneity in the RevMan software (RevMan, 2014; Cochrane). We reported the results of the fixed effects model unless we used the random effects model. We used the guide outlined in the Cochrane Handbook for Systematic Reviews of Interventions to interpret heterogeneity: ${ }^{12)} 0 \%$ to $40 \%$, might not be important; $30 \%$ to $60 \%$, might represent moderate heterogeneity; $50 \%$ to $90 \%$, might represent substantial heterogeneity; and $75 \%$ to $100 \%$, might indicate considerable heterogeneity.

\section{Grading Quality of Evidence}

We used the principles of the Grades of Recommendation, Assessment, Development, and Evaluation (GRADE) approach to evaluate the quality of the evidence in the systematic reviews. The GRADE approach is based on the study design, risk of bias, inconsistency, imprecision, and indirect evidence. We used the GRADEpro software to detect the quality of evidence for each individual outcome, and the assessment was collated with the "summary of findings" table.

\section{Ethics Approval and Consent to Participate}

An ethical statement is not applicable for this review and meta-analysis. Consent for publication is not applicable for this review and meta-analysis. The registration number of PROSPERO, an international database of prospectively registered systematic reviews, is CRD42019131602.

\section{RESULTS}

\section{Trial Selection}

We retrieved 164 records from the search of the electronic databases and 20 records from other sources (Figure 1). We screened 112 records, reviewed the full copies of 13 studies, and eight articles were identified as possibly meeting the review inclusion criteria, whereas the other five were not eligible for inclusion. Among the five trials excluded from this study, one had no control group, ${ }^{13)}$ whereas another was not an RCT trial and had a non-comparable population. ${ }^{14)}$ Another trial was written as a letter to the editor and did not report the outcomes of interest. ${ }^{15)}$ The other two excluded papers were an educational article on different types of galactagogues ${ }^{9)}$ and a systematic review and metaanalysis of the effect of metoclopramide on prolactin levels in breastfeeding mothers. ${ }^{16)}$ We finally included eight trials and excluded five trials from the present review.

\section{Characteristics of Included Trials}

Eight trials with 342 participants were included in our study. ${ }^{17-24)}$ Five of the eight trials (and four that contributed to the primary outcome) ${ }^{19-22)}$ declared funding and support from related institutions (Table 1). Five of the eight trials were conducted in high-income countries $^{17,18,20-22)}$ and three in middle-income countries. ${ }^{19,23,24)}$ Five of the eight trials recruited participants from healthcare settings and were all conducted at a single center. ${ }^{18,20-23)}$ Three trials did not mention the setting where the participants were recruited ${ }^{17,19,24)}$ and one was conducted at a neonatal intensive care unit. ${ }^{21)}$

Four trials reported the exclusion of participants because of acute illness, receiving medications that were contraindicated for breastfeeding or with metoclopramide use, a history of depression, women with breast-related disease (i.e., breast carcinoma), women with an anatomical abnormality of the breast, mothers with infants who had a congenital anomaly, and maternal age $<16$ years old. ${ }^{18,20,21,23)}$ Two trials consisted of 33 participants and involved only primipara mothers, ${ }^{17,23)}$ whereas in another study, $40 \%$ of the participants were primipara. ${ }^{24)}$ Three trials involved 175 mothers with preterm infants as their subjects of interest, ${ }^{18,20,21)}$ whereas 117 mothers with term infants were included in four reported trials. ${ }^{17,19,22,23)}$ One trial included both mothers with term and preterm infants. ${ }^{24)}$

The participants in the trials were randomized into intervention and control groups. In seven trials, the participants in the intervention groups received $10 \mathrm{mg}$ metoclopramide orally thrice daily ${ }^{17,18,20-24)}$ and

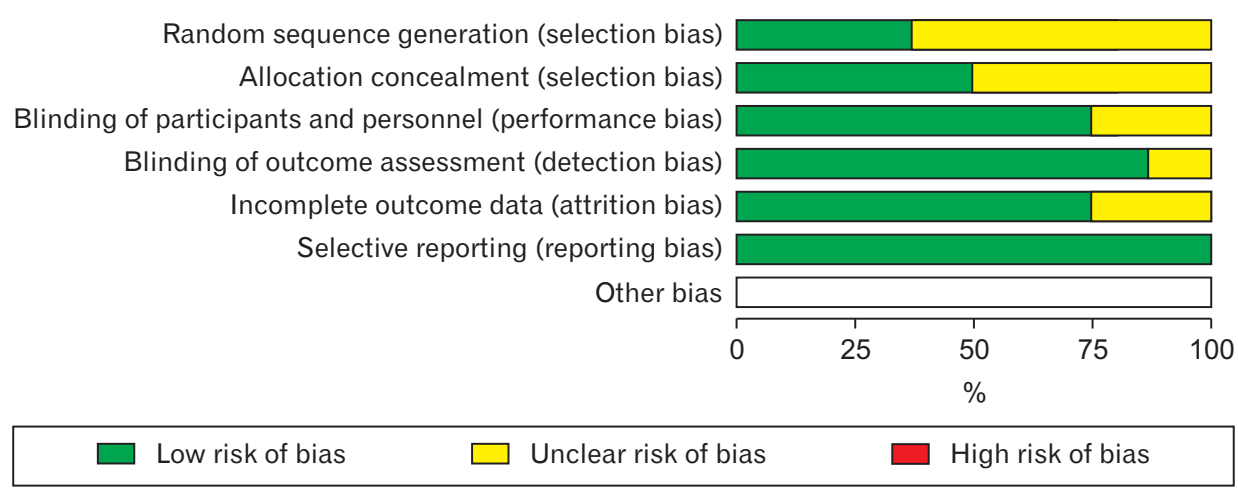

Random sequence generation (selection bias) Allocation concealment (selection bias) Blinding of outcome assessment (detection bias) Incomplete outcome data (attrition bias) Selective reporting (reporting bias) Other bias 0 Low risk of bias Unclear risk of bias High risk of bias
Figure 2. Risk of bias graph: a review based on authors' judgements about each risk of bias item presented as percentage across all included studies. 
in one trial, they were administered $10 \mathrm{mg}$ metoclopramide orally twice daily. ${ }^{19)}$ The duration of treatment for each trial varied. Two trials administered the intervention for 8 days, ${ }^{17,18)}$ three administered it for 10 days, ${ }^{20,21,24)}$ and one treated participants for 15 days. ${ }^{23)}$ One trial administered metoclopramide for 3 weeks ${ }^{22)}$ and another administered the intervention for 4 weeks. ${ }^{19)}$

\section{Risk of Bias Assessment}

The assessment of risk of bias is shown in Figure 2, which shows the proportion of studies assessed as having a low, high or unclear risk of bias for each risk of bias indicator and Figure 3, which shows the risk of bias indicators for the individual studies. The details of risk of bias as-

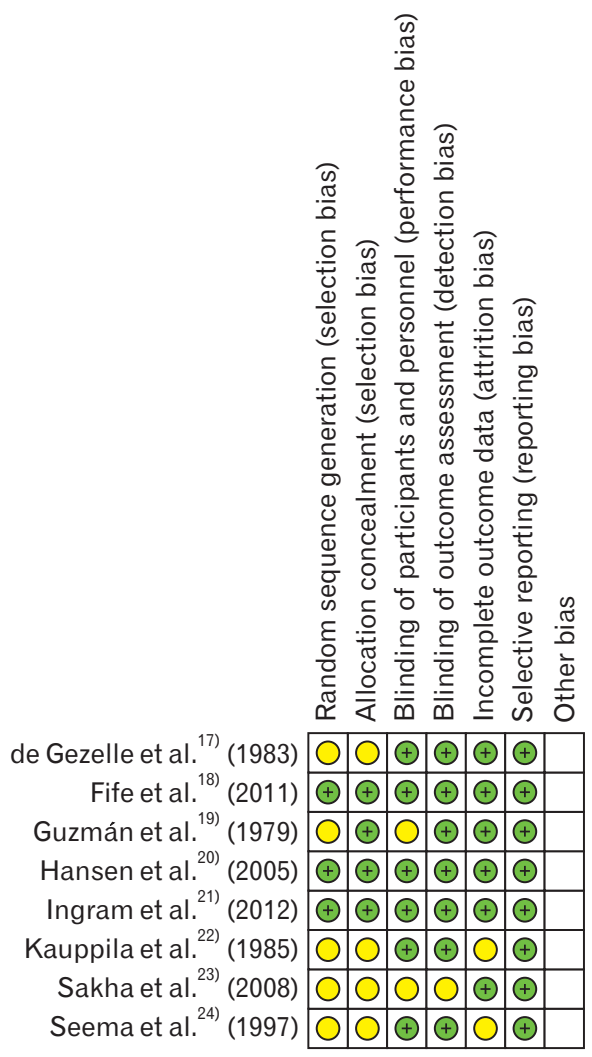

Figure 3. Summary of risk of bias: a review based on authors' judgements about each risk of bias item for each included study. sessment are presented in Appendix 1.

\section{Clinical Outcomes}

For the primary outcome, four trials reported milk production data in a usable format (four trials, 154 participants: MD, 10.21; 95\% CI, -10.16 to 30.57 ; fixed effects; $\mathrm{I}^{2}=84 \%$; low quality evidence) (Figure 4 , Table 2). ${ }^{17,18,20,21)}$ Three trials compared the effects of metoclopramide with those of the placebo on the mothers' serum prolactin levels at 2 weeks (three trials, 55 participants: MD, 216.79; 95\% CI, 186.58-247.01; fixed effects; $\mathrm{I}^{2}=82 \%$; moderate quality evidence) (Figure 5 , Table 2). ${ }^{17,19,22)}$

For the secondary outcome, infant weight gain was reported in two trials as an indirect effect of metoclopramide on milk production. ${ }^{24)}$ Milk intake was considered adequate based on several criteria, including adequate infant weight gain. There was no difference in the pattern of weight gain during the 14 weeks of the follow-up period. One trial provided the mean infant weight gain, ${ }^{23)}$ but we were unable to perform the meta-analysis because the standard deviation was not included. The mean weight gain of the intervention and control groups was $351.5 \mathrm{~g}$ and $328.5 \mathrm{~g}$, respectively over 15 days $(\mathrm{P}=0.68)$. Only one study addressed the duration of breastfeeding and a median (interquartile range) of 8.6 (5.6-16.9) weeks and 8.8 (3.4-12.0) weeks was recorded for the control and metoclopramide groups, respectively $(\mathrm{P}=0.093) .{ }^{20)}$

Maternal adverse events were reported in three trials. Headache was experienced by mothers in three trials (112 participants: RR, 1.33; 95\% CI, 0.44-4.01; fixed effects; $\mathrm{I}^{2}=0 \%$; moderate quality evidence) (Table 2 , Appendix 2). ${ }^{18,21,22)}$ Two trials recorded diarrhea as an adverse event (87 participants: RR, 1.75; 95\% CI, 0.33-27; fixed effects; $\mathrm{I}^{2}=47 \%$; moderate quality evidence) (Table 2, Appendix 3). ${ }^{18,21)}$ Fatigue was reported among the participants in two trials (47 participants: RR, 1.56; $95 \% \mathrm{CI}$, 1.04-2.35; fixed effects; $\mathrm{I}^{2}=0 \%$; moderate quality evidence) (Table 2, Appendix 4). ${ }^{18,22)}$

Dizziness was reported in two trials (90 participants: RR, 0.36; 95\% CI, 0.04-3.25; fixed effects; $\mathrm{I}^{2}=0 \%$; moderate quality evidence) (Table 2 , Appendix 5). ${ }^{21,22)}$ Three different trials, the participants reported symptoms of depression, constipation, and insomnia, ${ }^{18)}$ whereas nausea was reported in another trial. ${ }^{22)}$ Mood swings and dry mouth were documented in the trial. ${ }^{21)}$ For all outcomes, there was no substantial change in the effect size and CI after removing the trials with an un-

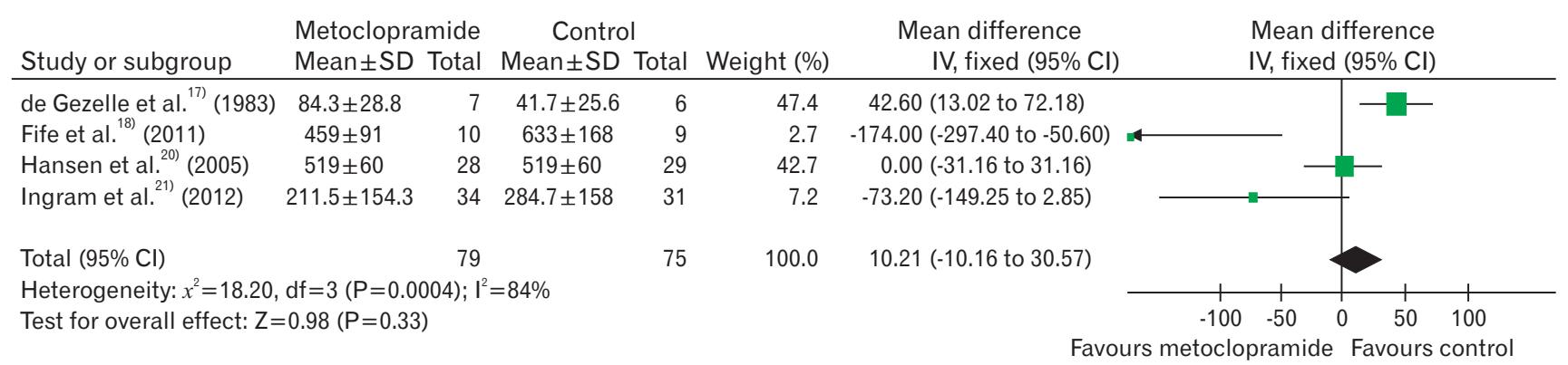

Figure 4. Milk production. SD, standard deviation; IV, inverse variance; Cl, confidence interval; df, degrees of freedom. 


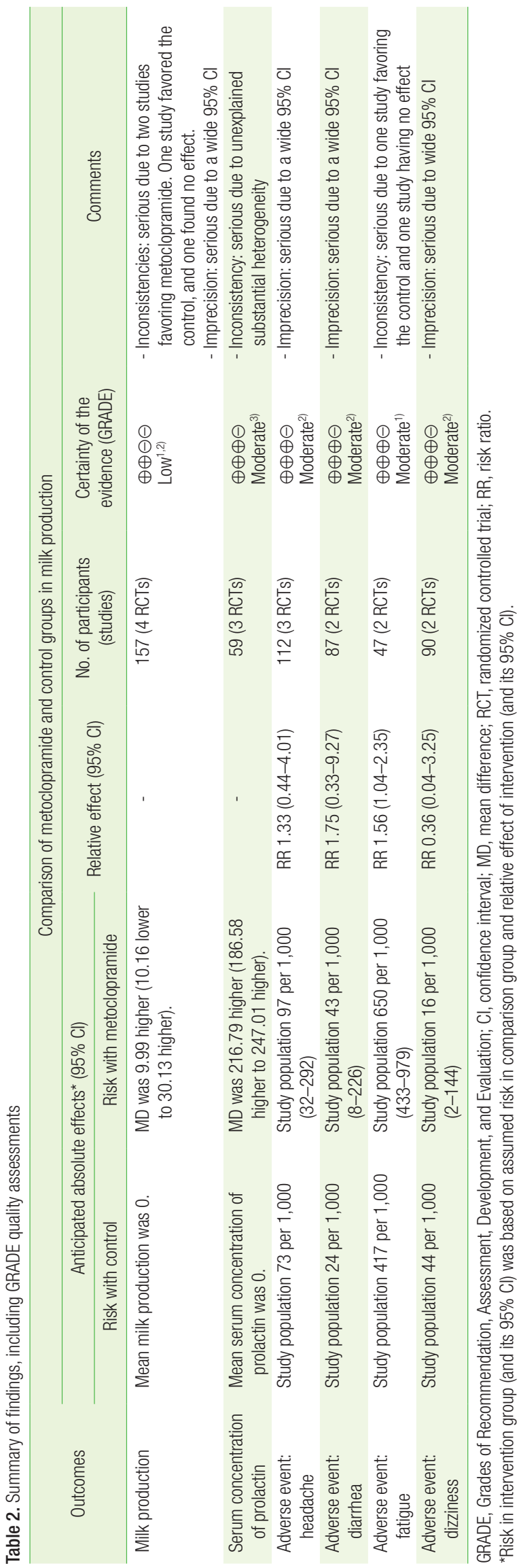

clear risk of bias for allocation concealment and random sequence generation.

\section{DISCUSSION}

All the RCTs included in this review reported the effectiveness of metoclopramide for milk production among women of reproductive age. This review showed that metoclopramide did not induce higher milk volumes in the intervention groups than those reported in the control groups. However, there was a significant increase in the serum concentrations of prolactin of mothers administered metoclopramide. No significant adverse events were reported. Recently, Foong et al. ${ }^{25)}$ reviewed the effect of oral pharmacological and natural galactagogues in increasing milk production. This review finally analyzed one study of metoclopramide with an outcome of infant weight where there was little or no difference with the control group, and three studies of the effects of metoclopramide, domperidone, and sulpiride on milk volume. ${ }^{25)}$ In contrast, our present review focused on metoclopramide to maintain homogeneity in the intervention for comparability with the controls.

\section{Overall Completeness and Applicability of Evidence}

We assessed the effectiveness of metoclopramide for milk production by extensively analyzing the existing literature. Eight trials were included in the review, but only six were included in the meta-analysis ${ }^{23)}$ and reported the mean value only; therefore, we could not calculate the standard deviation because of insufficient data. Moreover, a study only reported the outcomes figure (graph); ${ }^{24)}$ therefore, the exact data could not be assessed. Hansen et al. ${ }^{20)}$ reported primary (milk production) and secondary (duration of breastfeeding) outcomes.

However, the duration of breastfeeding data could not be assessed in the meta-analysis because only the median was provided, and this would have limit the applicability of the findings of this review. The incidences of adverse events were reported in three trials, and we concluded that four adverse events were common across the studies (i.e., headache, diarrhea, dizziness, and fatigue). However, only a few studies reported these adverse events, and the data were limited. Nevertheless, none of the adverse events were considered serious and rare adverse events, especially extrapyramidal reactions, were reported in the RCTs involving breastfeeding women.

\section{Quality of Evidence}

There were variations in the quality of the evidence and trials, although most of the domains across all the trials were of low or unclear risk. None of the trials published included protocols, but we concluded there was no selective reporting bias because all the outcomes listed in the objectives were reported in the full trial papers. Five studies demonstrated an unclear risk in random sequence generation, mostly due to the unexplained methods of randomization. Some of the studies also had an unclear risk of allocation, which could have led to treatment effect bias. The risk of attrition bias was identified in two trials, 


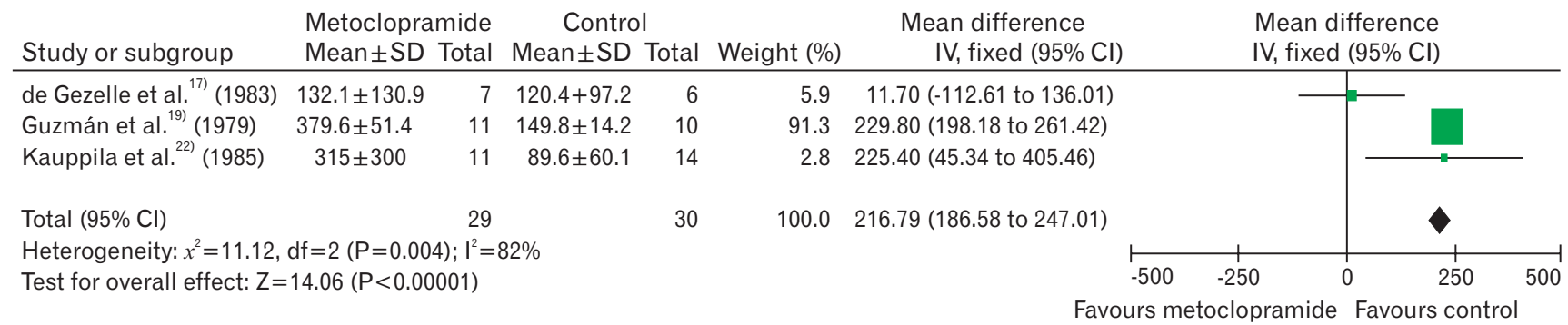

Figure 5. Concentration of serum prolactin. SD, standard deviation; IV, inverse variance; $\mathrm{Cl}$, confidence interval; df, degrees of freedom.

where the attrition bias was unclear due to the absence of explanations of the loss to follow-up. None of the trials conducted intention-to-treat analyses.

Five trials reported funding, including those that received funding from their respective institutions and breast pump manufacturers. The results of effects on milk production were derived from 154 mothers and showed substantial heterogeneity with a wide $95 \%$ CI. We performed additional sensitivity analyses using a few factors, such as mothers with preterm or term infants, primipara, and the time treatment started, which may have affected heterogeneity. However, the removal of any individual study did not affect the overall MD or $\mathrm{I}^{2}$ value. Although the outcome of serum prolactin levels of the mothers showed high heterogeneity across the studies and wide variations occurred in the standard deviations, the outcomes remained significant. A subgroup analysis also did not explain such effects.

Some heterogeneity was noted in the secondary outcome (occurrence of adverse events) among the studies but the sample size was small and number of studies was limited and, consequently, the sensitivity analysis did not reveal any differences. Because the heterogeneity was high, we used fixed effects analyses. Some of the secondary outcomes were not analyzed in the meta-analysis because of incomplete data and the results were reported as figures. ${ }^{23,24)}$ Therefore, we concluded that the overall quality of the evidence in this review was moderate to low.

\section{Potential Bias in Review Process}

We checked the references lists of all the studies and searched various databases for further references to reduce publication bias as much as possible. However, we cannot guarantee that all relevant trials were reviewed. We finally included eight trials, but could not assess publication bias, which remained undetected for all the studies. This was due to the limited number of small trials. Accordingly, there were insufficient studies for comparison, and the outcomes did not allow the use of funnel plots to detect bias. Not all the trials were included in the meta-analysis because of incomplete data. ${ }^{23)}$ Furthermore, a graph was used to present the outcomes of the trial in one article; ${ }^{24)}$ therefore, we could not obtain the exact data. Importantly, not all the included studies reported all the outcomes we needed for the analysis.

\section{Agreements and Disagreements with Other Studies and Reviews}

Only one review studied the effects of metoclopramide on maternal serum prolactin levels. ${ }^{16)}$ The study included RCTs, quasi-experimental clinical trials and cross-over trials, and five trials were included in the review. The authors excluded all the studies, which did not include comparison groups and those that evaluated other parameters, such as milk volumes and infant weight gain. Only three of five trials were included in our study because the remaining did not fulfil our study eligibility criteria and were not related to our outcomes. ${ }^{17,19,22)}$ A review examined studies that administered $10 \mathrm{mg}$ metoclopramide 3 times a day and measured the serum prolactin levels of mothers in both the second and third weeks. ${ }^{16)}$ Thus, the meta-analysis included data from both the second and third weeks. However, for our review, in the primary outcome analysis, we only included data from day 7 to 14 , which is the minimum time required to observe the peak drug effects. The review reported an increase in the serum prolactin levels of mothers in the third week, whereas the results of the second week of metoclopramide administration did not affect this parameter. ${ }^{16)}$ That study also delineated the side effects of metoclopramide, but a meta-analysis was not conducted.

\section{Conclusion}

This meta-analysis demonstrates that there is insufficient evidence to support the effectiveness of metoclopramide compared to other control treatments in increasing milk production in women who are breastfeeding their children. Numerous factors can enhance milk production and, therefore, we do not recommend the use of metoclopramide as a galactagogue in clinical practice. Although the included studies in this review reported that metoclopramide increased maternal serum prolactin concentration levels, it is noteworthy that prolactin levels can also be affected by infant suckling. Therefore, the findings may be overlapping. Accordingly, more research with larger sample sizes should be conducted in the future. Data on serious and rare adverse events were limited in this review, therefore, more data is needed to assess the safety of the drug.

Future studies should include larger sample sizes consisting of both term and preterm infants to compare these two groups and identify the differences between them, as well as any maternal or infant side effects and the outcomes measured. We did not provide details on the 
timing of the introduction of medication in our review, and this remains controversial as it could influence the effects of treatment on expressed breast milk production. Accordingly, future studies should focus on this issue.

\section{CONFLICT OF INTEREST}

No potential conflict of interest relevant to this article was reported.

\section{ACKNOWLEDGMENTS}

The authors would like to thank Amran Mamat and Nurul Azurah Mohd Roni, librarians, from Hamdan Tahir Library, for their assistance with database searches.

\section{ORCID}

Nik Hazlina Nik Hussain: https://orcid.org/0000-0002-9476-062X

Norhayati Mohd Noor: https://orcid.org/0000-0002-6372-1476

Shaiful Bahari Ismail: https://orcid.org/0000-0002-2000-2332

Nur Amirah Zainuddin: https://orcid.org/0000-0002-3794-3294

Zaharah Sulaiman: https://orcid.org/0000-0002-4023-1845

\section{REFERENCES}

1. Bhandari N, Kabir AK, Salam MA. Mainstreaming nutrition into maternal and child health programmes: scaling up of exclusive breastfeeding. Matern Child Nutr 2008;4(Suppl 1):5-23.

2. World Health Organization; United Nations Children's Fund. Global strategy for infant and young child feeding. Geneva: World Health Organization; 2003.

3. Amir LH. Managing common breastfeeding problems in the community. BMJ 2014;348:g2954.

4. Stuebe AM. Enabling women to achieve their breastfeeding goals. Obstet Gynecol 2014;123:643-52.

5. Wagner EA, Chantry CJ, Dewey KG, Nommsen-Rivers LA. Breastfeeding concerns at 3 and 7 days postpartum and feeding status at 2 months. Pediatrics 2013;132:e865-75.

6. Powers NG. Slow weight gain and low milk supply in the breastfeeding dyad. Clin Perinatol 1999;26:399-430.

7. Amir LH. Breastfeeding: managing 'supply' difficulties. Aust Fam Physician 2006;35:686-9.

8. Academy Of Breastfeeding Medicine Protocol Committee. ABM Clinical Protocol \#9: use of galactogogues in initiating or augmenting the rate of maternal milk secretion (first revision January 2011). Breastfeed Med 2011;6:41-9.
9. Gabay MP. Galactogogues: medications that induce lactation. J Hum Lact 2002;18:274-9.

10. Sousa PL. Letter: Metoclopramide and breast-feeding. Br Med J 1975;1:512.

11. Anderson PO. The galactogogue bandwagon. J Hum Lact 2013;29:710.

12. Higgins JP, Altman DG, Gotzsche PC, Juni P, Moher D, Oxman AD, et al. The Cochrane Collaboration's tool for assessing risk of bias in randomised trials. BMJ 2011;343:d5928.

13. Ehrenkranz RA, Ackerman BA. Metoclopramide effect on faltering milk production by mothers of premature infants. Pediatrics 1986;78: 614-20.

14. Toppare MF, Laleli Y, Senses DA, Kitapci F, Kaya IS, Dilmen U. Metoclopramide for breast milk production. Nutr Res 1994;14:1019-29.

15. Lewis PJ, Devenish C, Kahn C. Controlled trial of metoclopramide in the initiation of breast feeding. Br J Clin Pharmacol 1980;9:217-9.

16. Osouli Tabrizi S, Mirghafourvand M, Seyedi R. The effect of metoclopramide on prolactin levels in breastfeeding mothers: a systematic review and meta-analysis. Int J Pediatr 2017;5:5827-38.

17. de Gezelle H, Ooghe W, Thiery M, Dhont M. Metoclopramide and breast milk. Eur J Obstet Gynecol Reprod Biol 1983;15:31-6.

18. Fife S, Gill P, Hopkins M, Angello C, Boswell S, Nelson KM. Metoclopramide to augment lactation, does it work?: a randomized trial. J Matern Fetal Neonatal Med 2011;24:1317-20.

19. Guzman V, Toscano G, Canales ES, Zarate A. Improvement of defective lactation by using oral metoclopramide. Acta Obstet Gynecol Scand 1979;58:53-5.

20. Hansen WF, McAndrew S, Harris K, Zimmerman MB. Metoclopramide effect on breastfeeding the preterm infant: a randomized trial. Obstet Gynecol 2005;105:383-9.

21. Ingram J, Taylor H, Churchill C, Pike A, Greenwood R. Metoclopramide or domperidone for increasing maternal breast milk output: a randomised controlled trial. Arch Dis Child Fetal Neonatal Ed 2012; 97:F241-5.

22. Kauppila A, Anunti P, Kivinen S, Koivisto M, Ruokonen A. Metoclopramide and breast feeding: efficacy and anterior pituitary responses of the mother and the child. Eur J Obstet Gynecol Reprod Biol 1985;19: 19-22.

23. Sakha K, Behbahan AG. Training for perfect breastfeeding or metoclopramide: which one can promote lactation in nursing mothers? Breastfeed Med 2008;3:120-3.

24. Seema, Patwari AK, Satyanarayana L. Relactation: an effective intervention to promote exclusive breastfeeding. J Trop Pediatr 1997;43: 213-6.

25. Foong SC, Tan ML, Foong WC, Marasco LA, Ho JJ, Ong JH. Oral galactagogues (natural therapies or drugs) for increasing breast milk production in mothers of non-hospitalised term infants. Cochrane Database Syst Rev 2020;5:CD011505. 


\section{Appendix 1. Risk of bias assessment}

\section{Allocation}

Four trials described the method of randomisation used. Of these, two trials randomised the participants using computer-generated randomisation, ${ }^{18,20)}$ and one trial applied allocations based on random number tables. ${ }^{21)}$ One trial applied a simple random sampling procedure. ${ }^{24)}$ However, this sequence of assignment can be unpredictable, and it was not described further, hence it was unclear. The method of randomisation was not reported in the other four trials, and we therefore judged random sequence generation as an unclear risk in four studies. ${ }^{17,19,22,23)}$ Allocation concealment was not clear in four trials. ${ }^{17,22-24)}$

\section{Blinding}

Five trials used a placebo control. ${ }^{17-20,22)}$ Although one trial gave a placebo to the control group ${ }^{23)}$ the blinding was not described gave the control group domperidone, ${ }^{21)}$ but the medication was kept in a gelatine capsule, and both the participants and personnel were blinded to which drug they took. One trial gave both groups vitamin supplements in addition to the drug given to the intervention group. ${ }^{24)}$

\section{Incomplete Outcomes Data}

Six trials reported the primary outcomes, ${ }^{17-22)}$ but only four were included in the meta-analysis. ${ }^{17,18,20,21)}$ Four studies provided details of the participants who were excluded or withdrew from the study during follow-up. ${ }^{18,20-22)}$ However, the attrition bias was considered low in three studies because the reasons for exclusion and withdrawal were stated..$^{18,20,21)}$ The missing data were balanced across the groups and were not related to the outcomes. One trial was considered to have an unclear risk of attrition bias because the reasons for the withdrawals were not mentioned. ${ }^{22)}$ Six trials included the secondary outcomes. ${ }^{18,20-24)}$ However, three trials were not included in the meta-analysis, and two of them had a low risk of attrition bias. Reasons were provided for the withdrawals, and these were balanced across the groups ${ }^{20)}$ There were no withdrawals in one trial. ${ }^{23)}$ One trial reported the outcomes as median values, ${ }^{20)}$ while another revealed the mean only. ${ }^{23)}$ We therefore could not calculate the standard deviation due to incomplete data. Another study reported the data in figures (via a graph), ${ }^{24)}$ so we could not extract the exact data. None of the trials indicated the intention to treat the analyses.

\section{Selective Reporting}

All eight trials reported the outcomes as stated in their methods sections. ${ }^{17-24)}$

\section{Other Potential Sources of Bias}

No other potential sources of bias were detected.

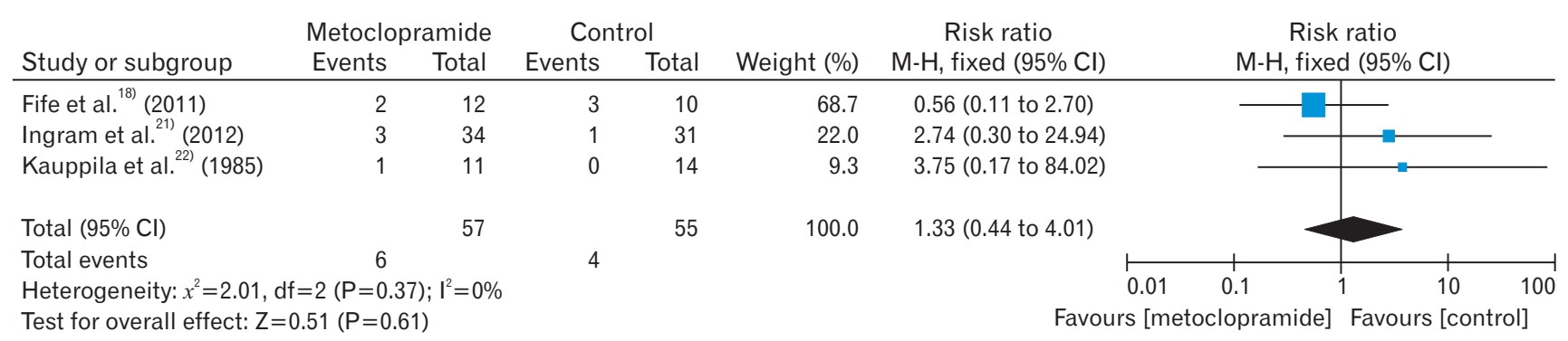

Appendix 2. Headache. M-H, Mantel-Haenszel; Cl, confidence interval; df, degrees of freedom. 


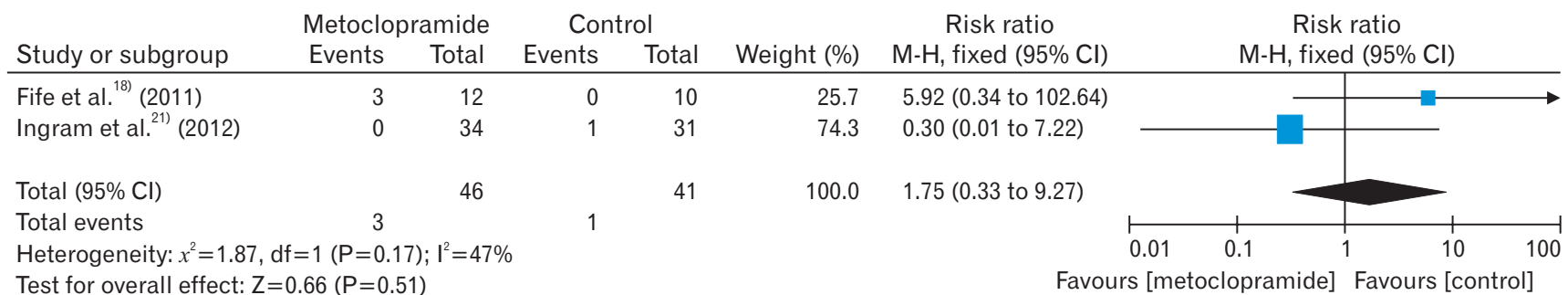

Appendix 3. Diarrhoea. M-H, Mantel-Haenszel; Cl, confidence interval; df, degrees of freedom.

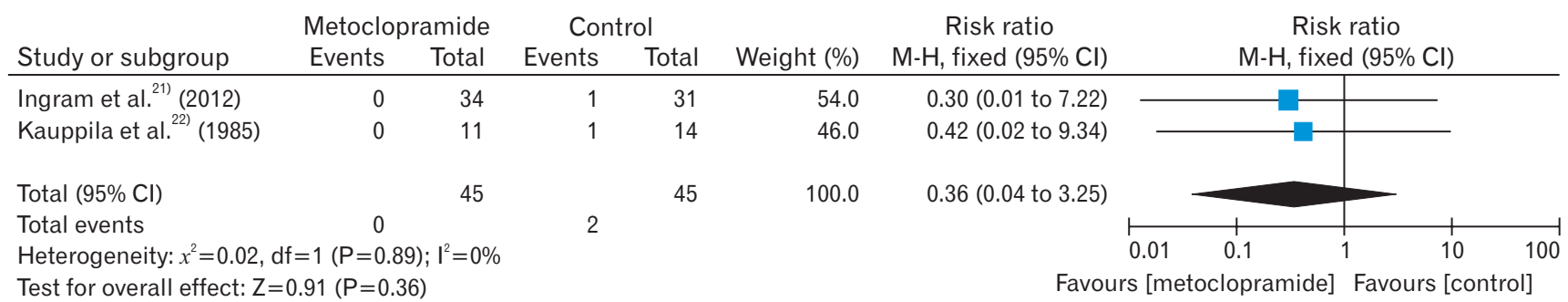

Appendix 4. Fatigue. M-H, Mantel-Haenszel; Cl, confidence interval; df, degrees of freedom.

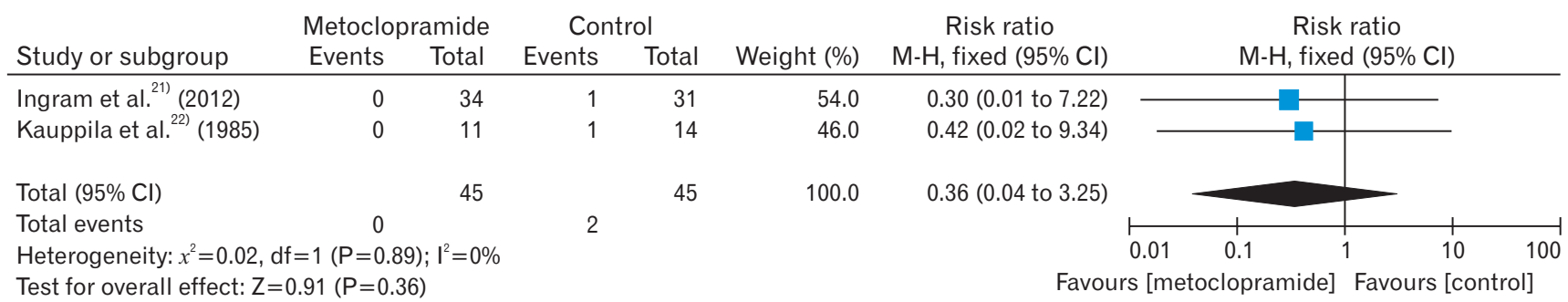

Appendix 5. Dizziness. M-H, Mantel-Haenszel; Cl, confidence interval; df, degrees of freedom. 